\title{
Ultra-Bright Fluorescent Organic Nanoparticles Based on Small-Molecule Ionic Isolation Lattices
}

\author{
Junsheng Chen, ${ }^{a}$ S. M. Ali Fateminia, ${ }^{a}$ Laura Kacenauskaite, ${ }^{a}$ Nicolai Bærentsen, ${ }^{a}$ \\ Stine Grønfeldt Stenspil, ${ }^{a}$ Jona Bredehoeft, ${ }^{a}$ Karen L. Martinez, ${ }^{a}$ Amar H. Flood, ${ }^{* b}$ Bo W. Laursen ${ }^{\star a}$
}

[a] Dr. J. Chen, Dr. S. Fateminia, Dr. L. Kacenauskaite, N. Bærentsen, S. Stenspil, J. Bredehoeft, Prof. K. Martinez, Prof. B. Laursen

Nano-Science Center \& Department of Chemistry, University of Copenhagen

Universitetsparken 5, 2100 København Ø, Denmark

E-mail: bwl@chem.ku.dk

[b] Prof. A. Flood

Molecular Materials Design Lab, Department of Chemistry, Indiana University

800 East Kirkwood Avenue, Bloomington, Indiana 47405, United States

E-mail: aflood@indiana.edu

\begin{abstract}
Ultra-bright fluorescent nanoparticles hold great promise for demanding bioimaging applications. Recently, extremely bright molecular crystals of cationic fluorophores were obtained by hierarchical co-assembly with cyanostar anion-receptor complexes of associated counterions. These small-molecule ionic isolation lattices (SMILES) ensure spatial and electronic isolation to prohibit dye aggregation quenching. We report a simple, one-step supramolecular approach to formulate SMILES materials into nanoparticles. Rhodamine-based SMILES nanoparticles stabilized by glycol amphiphiles show high fluorescence quantum yield (30\%) and brightness per volume $\left(5000 \mathrm{M}^{-1} \mathrm{~cm}^{-1} / \mathrm{nm}^{3}\right)$ with 400 dyes packed into 16-nm particles, corresponding to an absorption coefficient of $4 x$ $10^{7} \mathrm{M}^{-1} \mathrm{~cm}^{-1}$. UV excitation of the cyanostar component leads to highest brightness $\left(>6000 \mathrm{M}^{-1} \mathrm{~cm}^{-1} / \mathrm{nm}^{3}\right)$ by energy transfer to rhodamine emitters. Coated nanoparticles stain cells and are thus promising for bioimaging.
\end{abstract}

\section{Introduction}

Fluorescent materials are a prerequisite for a wide range of technologies including displays, lasers, sensors, diagnostic assays, and bioimaging reagents; ${ }^{[1]}$ and their development is a fundamental and highly active area of chemistry and materials science. To these ends, use of organic molecular emitters in the construction of fluorescent materials, particularly nanoparticles (NPs), is attractive. Molecular fluorophores offer widely tunable optical properties, as dictated by their covalent structure, and they circumvent use of toxic elements ${ }^{[2]}$ that often accompany inorganic nanoparticles. ${ }^{[3]}$

A general parameter needed for the optimization of emissive materials is the brightness $(B)$. In molecular materials, brightness is a product of the material's ability to absorb the excitation light (molar absorption coefficient, $\varepsilon$ ) and the ability of the material to emit light (fluorescence quantum yield, $\phi$ ): $B=\varepsilon \times \phi$. In organic fluorescent nanoparticles composed of molecular fluorophores, the brightness can be defined by the number $(n)$ of dyes in the particles: $B=n \times \varepsilon \times \phi$. Theoretically, therefore, the brightness of such nanoparticles, with $n>100$, can be orders of magnitude higher than a single fluorescent dye. However, when considering possible applications in bioimaging, the drive towards a brighter (and larger) particle is opposed by the preference for smaller particles. ${ }^{[4]}$ Therefore, the brightness per unit volume
$(B / V)$ is used to address the brightness independently of the particle size and to compare material performance across various embodiments. ${ }^{[5]}$ To achieve high normalized brightness, $B / V$, a high density of fluorescent dyes is required inside the nanoparticles. However, when typical fluorescent dyes are packed closely together within a nanostructure, excitonic coupling between them leads to spectral shifts and quenching of emission with substantial losses in both light absorption $(\varepsilon)$ and emission $(\phi)$. This ubiquitous effect, known as aggregation-caused quenching, prohibits the direct translation of optical properties of the most efficient fluorescent dyes from solution to densely packed solid-state materials. ${ }^{[6]}$

Various strategies have been proposed to improve the brightness of organic fluorescent nanoparticles. However, it is a tall order to increase the loading of fluorescent dyes while also being constrained by aggregation-caused quenching. ${ }^{[5,7]}$ The standard approach is to introduce space filling groups, such as covalently introducing bulky side groups to fluorescent dyes using timeconsuming synthesis ${ }^{[8]}$ and dilution in polymers, ${ }^{[9]}$ that nevertheless render formulation as a hit-or-miss activity. Separation of fluorescent dyes can also be realized in metal organic frameworks, ${ }^{[10]}$ ionic liquids, ${ }^{[1]}$ DNA scaffolds, ${ }^{[12]}$ or silica nanoparticles. ${ }^{[13]}$ Another approach is by aggregation-induced emission, ${ }^{[14]}$ in which fluorescence is obtained when flexible dyes with bulky and contorted structures are constrained in solids. ${ }^{[15]}$ Successful particle preparation must also provide a means to retain fluorescence without introducing losses resulting from the surface states present in high abundance relative to bulk on the nanometer length scale. Successful approaches to bright organic fluorescent nanoparticles were developed by Reisch and Klymchenko based on polymer nanoparticles loaded with high concentrations of cationic dyes paired with large (space-filling) fluorinated counterions. ${ }^{[16]}$

Recently we discovered a simple and effective approach to transfer the optical properties of typical cationic dyes directly and reliably from solution to high-density molecular crystals. ${ }^{[17]}$ In this case, addition of cyanostar (CS) macrocycles serving as an anion receptor to assemble around the counter anions, also enforces charge-by-charge alternating packing to isolate the dye molecules. We call these crystalline molecular materials small-molecule ionic-isolation lattices (SMILES, Fig. 1). In SMILES crystals, the spatially and electronically isolated dyes preserve the attractive optical properties seen in dilute solution. We found that fluorescent SMILES crystals are formed by representatives of the 
most important classes of cationic dyes. These dyes included cyanines, oxazines, trianguleniums, and rhodamines. All of them provided predictable spectral properties, highly improved fluorescence quantum yields, and high densities of dyes (one dye per $\approx 4 \mathrm{~nm}^{3}$ ) leading to very high normalized brightness per volume $\left(>7000 \mathrm{M}^{-1} \mathrm{~cm}^{-1} / \mathrm{nm}^{3}\right)$ surpassing all other organic dyebased fluorescent materials. ${ }^{[5,17]}$ (a)

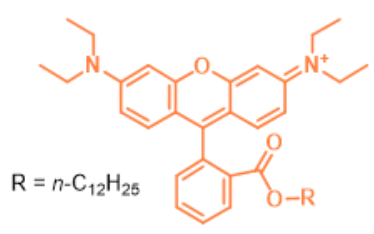

R12 (b)

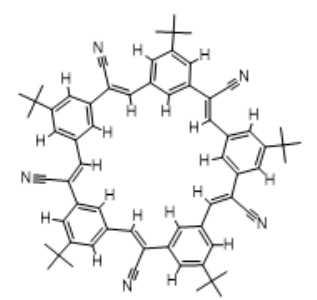

(c)

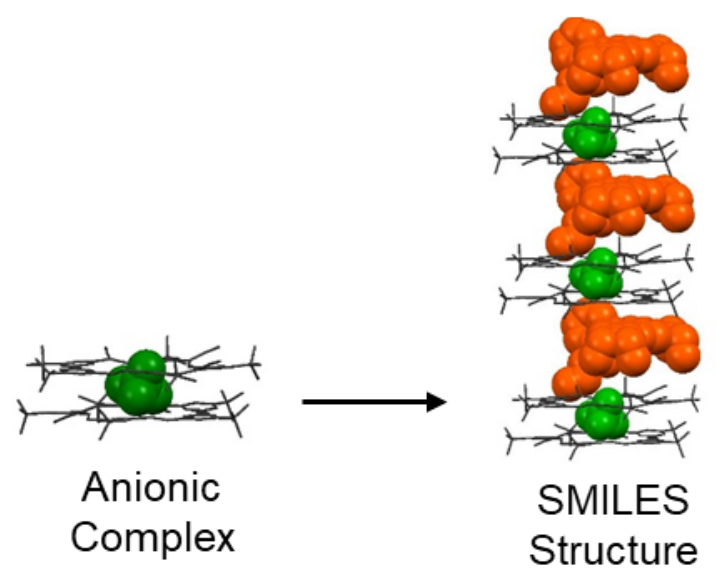

Figure 1. Illustration of the self-assembly process and the structure of small-molecule ionic isolation lattices (SMILES) materials. The molecular structure of lipophilic rhodamine R12 (a) and cyanostar anion receptor (CS) (b), which binds the $\mathrm{PF}_{6}{ }^{-}$ion in a 2-to-1 anionic complex (c). SMILES are produced by crystallization of the cationic dye with the anion complex (d). Space filling structures are from the reported single crystal structure of rhodamine $3 \mathrm{~B} \cdot \mathrm{CIO}{ }_{4} \mathrm{SMILES}(\mathrm{CCDC} 1892437)$.

With SMILES materials displaying these highly favorable properties available, we were motivated to investigate if the supramolecular SMILES concept could be transferred to nanoparticles without loss in performance. If possible, then the most logical potential application would be for bioimaging, which also motivated an exploration of a method to make them stable in aqueous solution. Herein we show that a simple method based on antisolvent nanoprecipitation can be used for the hierarchical assembly of SMILES nanoparticles from a rhodamine dye. We verified that the highly attractive optical properties of macroscopic crystals and thin films of SMILES materials can be transferred to 25-nm brightly fluorescent nanoparticles. We also discovered that the cyanostar-anion complex is multi-functional. In addition to directing the molecular packing for spatial and electronic isolation, it acts as an antenna to funnel UV excitation to the rhodamine dye, thereby giving rise to even higher brightness when the particles are excited in the UV region. By using amphiphilic surface coatings to confer compatibility with aqueous and biological media, we were able to produce long-term colloidal stability, and to fortuitously make the size smaller as well as improve the size distribution (16 $\pm 5 \mathrm{~nm}$ ). As a proof-of-concept, we also demonstrated the use of these SMILES nanoparticles as new tools for live cell bioimaging thereby emphasizing their utility and taking one step towards fulfilling the promise of ultra-bright nanoparticles.

\section{Results and Discussion}

\section{Formulation of SMILES Nanoparticles.}

We undertook the preparation of SMILES-based nanoparticles to first establish that they could be formed in aqueous solution and would retain the optical properties of the macroscale materials. For this reason, the neat and uncoated SMILES nanoparticles were prepared and studied first. In order to prepare SMILES nanoparticles that will avoid leaching of the dye component into the surrounding aqueous solution, we followed the strategy of Klymchenko and coworkers to modify commercially available rhodamine $B$ with a longer hydrophobic alkyl chain, ${ }^{[18]}$ in our case the dodecyl derivative called R12 (Fig. 1 and Fig. S1). A nanoparticle precursor solution was prepared in tetrahydrofuran (THF) and composed of the components needed to form SMILES materials, the cationic dye $\mathbf{R} 12$ as a salt of the $\mathrm{PF}_{6}{ }^{-}$counterion and the cyanostar macrocycle (see details in Section S2). The SMILES nanoparticles were formed by injecting this precursor solution $(520 \mu \mathrm{L})$ into a large excess of water $(10 \mathrm{~mL})$ under sonication for 1 minute (Fig. 2a). As a control, similar R12 THF solutions but with the lattice-forming cyanostar omitted were also subjected to the same nanoprecipitation method. The resulting colloids were only metastable and displayed aggregated structures labeled hereafter as R12 aggregates. 
(a)

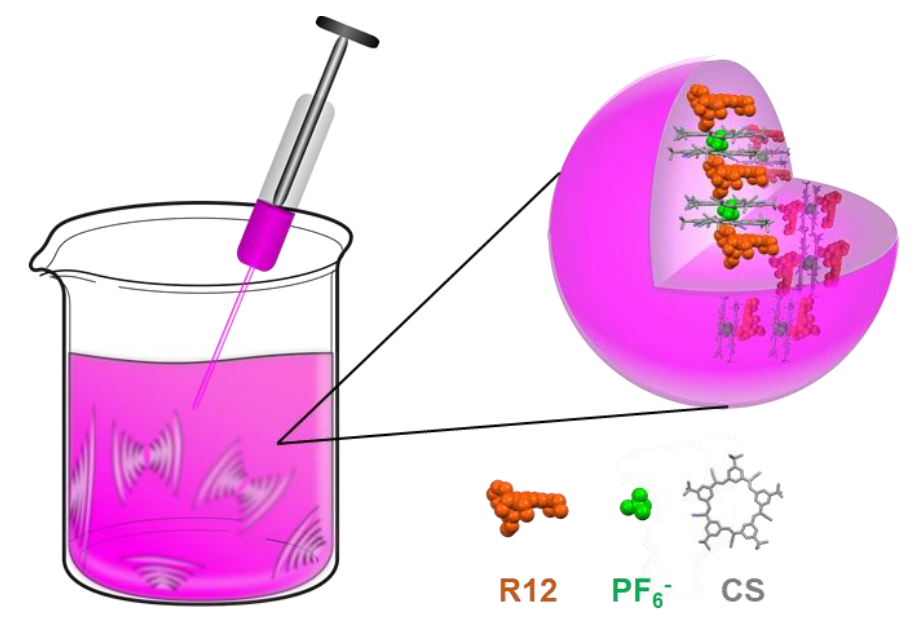

(b)

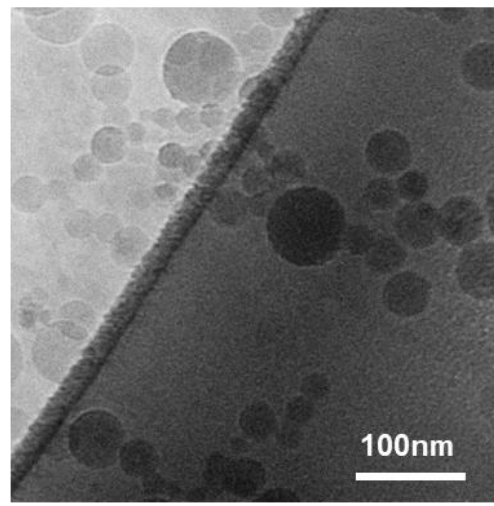

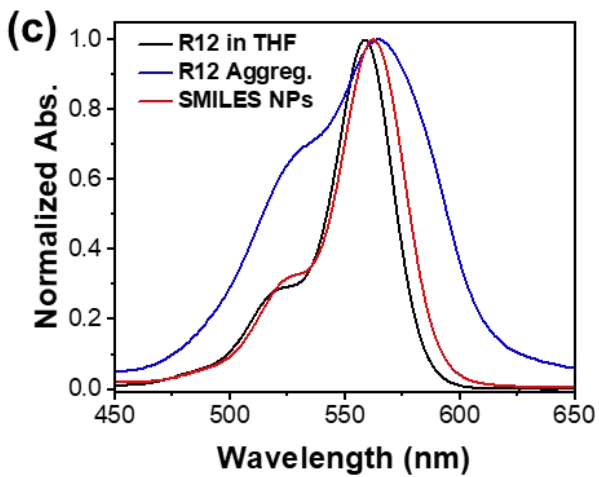

(d)

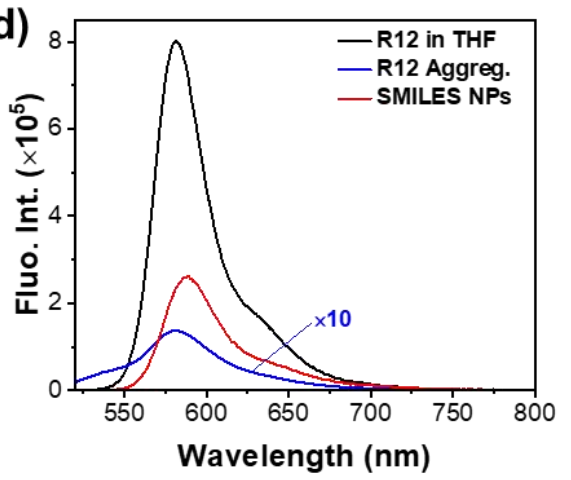

(e)

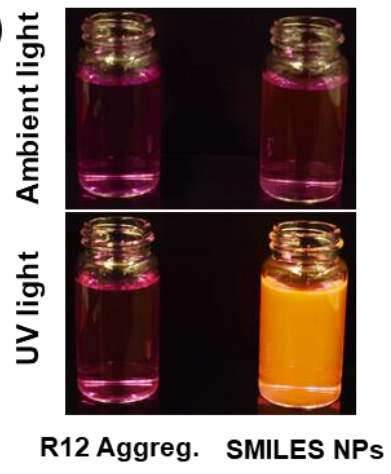

Figure 2. (a) Schematic representation of the preparation of SMILES nanoparticles (NPS) and a model of their internal structure; (b) Cryo-TEM image of SMILES nanoparticles made with 2.5 eq cyanostar; (c) Normalized absorbance of R12 in THF solution, in water as the R12 aggregates (Aggreg.) and in SMILES nanoparticles; (d) Absorption-normalized fluorescence spectra of R12 in THF solution, in water as R12 aggregates and in SMILES nanoparticles $\left(\lambda_{\text {ex }}=510\right.$ nm); (e) photos of R12 aggregates (formed without CS) and SMILES nanoparticles, upper panel: under ambient light; lower panel: under UV light irritation.

The successful formation of SMILES nanoparticles was indicated by a red transparent and highly fluorescent aqueous solution. This behavior contrasts with the control sample without cyanostar which was non-fluorescent (Fig. 2e). Cryogenic transmission electron microscopy (cryo-TEM) studies showed that the uncoated SMILES nanoparticles have a spherical morphology with a broad size distribution ( $25 \pm 8 \mathrm{~nm}$, see Fig. $2 \mathrm{~b}$ ), which was further confirmed by dynamic light scattering (DLS, see Fig. S2). High fidelity reproduction of the rhodamine's solution-phase optical properties was confirmed by absorption and fluorescence studies (Fig. 2c and 2d). When SMILES nanoparticle solutions were made by injecting the R12 precursor solution made with 2.5 molar equivalents cyanostar into water, the obtained aqueous solution shows similar absorption and emission spectra as a dilute solution of the R12 dye dissolved in THF. However, when a solution of the $\mathbf{R} 12$ dye alone was injected into water, the resulting aqueous solution shows a broadened and red-shifted absorption and weak fluorescence emission. These are typical features of dye aggregation and the fluorescence quenching that typically ensues. ${ }^{[19]}$ These studies confirm that the $\mathbf{R} 12$ dyes in the
SMILES nanoparticles have been electronically decoupled from each other upon addition of cyanostar. In other words, the R12SMILES nanoparticles display characteristics of the spatial and electronic isolation imposed by the hierarchically organized structure in SMILES materials.

The degree of crystalline order in the R12-SMILES nanoparticles was studied by collecting $x$-ray diffraction patterns from dried SMILES nanoparticles and comparing them with that of large SMILES crystals formed in organic solvents with R12. No specific scattering peaks were produced by the SMILES nanoparticle sample indicating a lack of long-range order (Fig. S3). The lack of a crystalline structure is not surprising, considering the use of fast precipitation during production of the nanoparticles. Nevertheless, the optical properties of these SMILES nanoparticles (Fig. 2) confirm that the hierarchical assembly of the alternating dyes and anion complexes locally produce an isolation lattice in accordance with crystalline SMILES materials (Fig. 1). Therefore, their noncrystalline structure is expected to have a negligible impact on the use of SMILES in nanoparticles. 
(a)

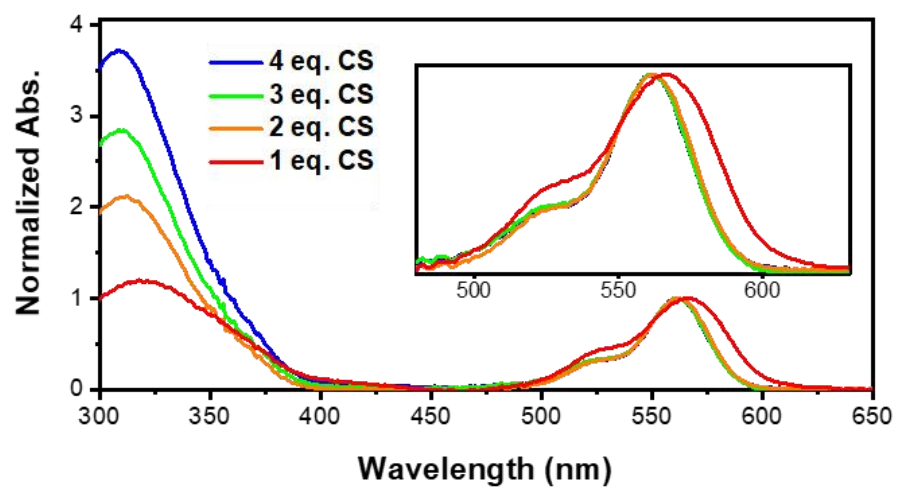

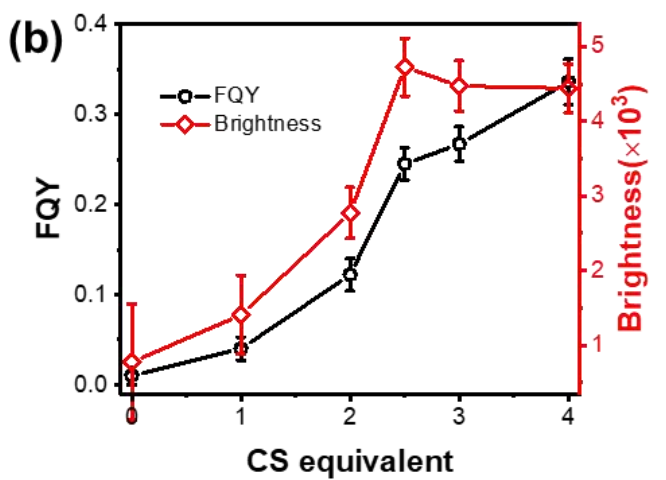

(d)

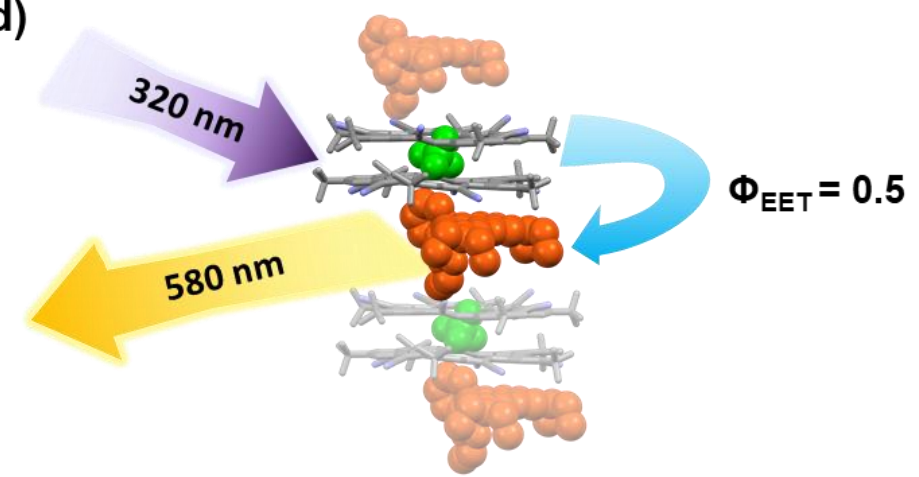

Figure 3. The role of cyanostar (CS) in SMILES R12 nanoparticles: (a) Absorption spectra as function of added cyanostar in R12 SMILES nanoparticles formed with different molar equivalents of cyanostar (1-4 equiv.) and no capping agent in aqueous solution. (b) Fluorescence quantum yield (FQY) and brightness per volume $(B / V)$ of neat SMILES nanoparticles with different molar equivalents of cyanostar added (excitation at $510 \mathrm{~nm}$ ); (c) Absorption and excitation (detection at $585 \mathrm{~nm}$ ) spectra of SMILES nanoparticles with 2.5 molar equivalent of cyanostar. (d) Schematic of the excitation energy transfer (EET) from cyanostar to R12 when SMILES nanoparticles are excited at $320 \mathrm{~nm}$.

\section{Multiple Functions of the Cyanostar Macrocycle in SMILES Nanoparticles.}

The functional role of cyanostar in the formation of ultra-bright SMILES nanoparticles was studied by varying the molar equivalents of cyanostar added to the precursor solution. It is clearly seen that the solution-like spectral properties, which is a signature of SMILES structures, ${ }^{[17]}$ are obtained once the amount of added cyanostar reaches 2 molar equivalents (Figs. 3a, S5). This value matches perfectly with the equivalence point for the stoichiometric reaction to form the 2:1 cyanostar: $\mathrm{PF}_{6}{ }^{-}$complex. The fluorescence quantum yield was seen (Fig. $3 \mathrm{~b}$ ) to increase 10 -fold with 2 equivalents of cyanostar $(\phi=11 \%)$ compared to R12 aggregates with 0 equivalents of cyanostar $(\phi=1 \%)$. Beyond 2 equivalents of the added cyanostar, the fluorescence quantum yield keeps increasing, reaching $34 \%$ at 4 equivalents (Fig. 3b and Table S3). The continuous increase in fluorescence quantum yield beyond 2 equivalents of cyanostar was assigned to the positive impact of the excess macrocycle on the passivation of defects that quench the fluorescence. Such defects are accessible by energy migration within the densely packed SMILES nanoparticles. ${ }^{[17,20]}$ However, the volume-normalized brightness of SMILES nanoparticles levels off beyond 2.5 molar equivalents of cyanostar, as the presence of excess cyanostar macrocycle in the nanoparticles also reduces the dye density. The brightness per volume $(B / V)$ of the R12-SMILES nanoparticles when excited in the main rhodamine absorption peak at $560 \mathrm{~nm}$ reaches a maximum of $4,725 \mathrm{M}^{-1} \mathrm{~cm}^{-1} / \mathrm{nm}^{3}$ with 2.5 molar equivalents of cyanostar (Fig. $3 \mathrm{~b}$ and Table S3). This is a substantial increase in performance relative to value seen in the particles of R12 alone $\left(\sim 800 \mathrm{M}^{-1} \mathrm{~cm}^{-1} / \mathrm{nm}^{3}\right)$ simply by adding cyanostars.

In a totally new finding, we discovered that the cyanostar macrocycles not only behave as integral structure-directing spacer units, but they also act as antennae by sensitizing the rhodamine dyes to UV light. The cyanostar macrocycles have a large molar absorption coefficient in the $300-350 \mathrm{~nm}$ region (Fig. 3a) where the R12 dye has negligible absorption (Fig S6). We find that the energy absorbed in the UV region by the cyanostar macrocycles within the SMILES lattice can be efficiently funneled to the fluorescent dyes. This behavior is seen in the excitation spectrum (Fig 3c) generated by detecting the emission from the R12 dye in the SMILES nanoparticles (see also Fig. S6 and S7). The energy transfer efficiency can be estimated from the intensity ratio of the excitation and absorption spectra for the cyanostar band at $320 \mathrm{~nm}$ by normalizing the two spectra to the $560 \mathrm{~nm}$ peak of the $\mathbf{R} 12$ dye (Fig. 3c). This ratio decreases as the loading of cyanostar macrocycles increases (Fig. S7). This finding agrees with the expectation that the excess cyanostar macrocycles are located further away from the R12 dyes within the SMILES nanoparticles, and thus they will transfer their excitation energy 
less efficiently to the R12 dyes. Parts of the excess cyanostar macrocycles may be accommodated in triple stacked anion complexes. ${ }^{[21]}$ The energy transfer efficiency $\left(\Phi_{\mathrm{EET}}\right)$ for SMILES nanoparticles with 2-3 molar equivalents of cyanostar is close to $50 \%$ (Figs. S7c and 3d). This outcome leads to a similarly halved fluorescence quantum yield when exciting the SMILES nanoparticles into the cyanostar-based absorption band at $320 \mathrm{~nm}$ compared with exciting R12 directly at $510 \mathrm{~nm}$ (see Tables S3 and S4). However, owing to the much larger absorption coefficient of cyanostar $\left(\varepsilon^{\sim} 10^{5} \mathrm{M}^{-1} \mathrm{~cm}^{-1}\right)$, the brightness of SMILES nanoparticles obtained using $320 \mathrm{~nm}$ excitation is still significantly higher when compared to direct excitation and can reach close to $10,000 \mathrm{M}^{-1} \mathrm{~cm}^{-1} / \mathrm{nm}^{3}$ for nanoparticles made with 4 equivalents of cyanostar ( $\phi=0.16$, see Table S4).

In this first translation of SMILES materials from macroscopic molecular crystals, thin films and polymer blends ${ }^{[17]}$ to a new molecular nanomaterial, we find that cyanostar molecules have multiple functions in SMILES nanoparticles. They act as a structure-directing spacer between the cationic dyes to prevent aggregation-caused quenching and constitute a UV excitation antenna system to boost the brightness, while excess cyanostar passivates quenching sites in the SMILES nanoparticles to raise the overall fluorescence quantum yield of the dye components.

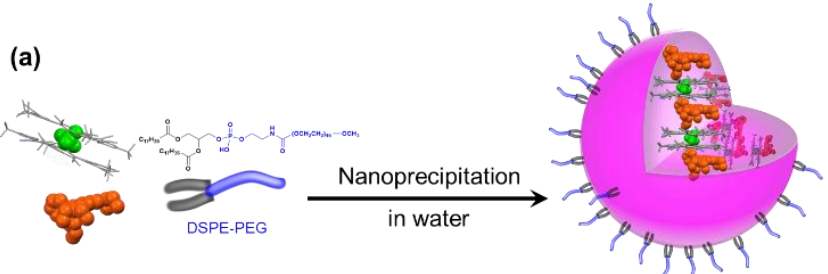

Precursors in THF

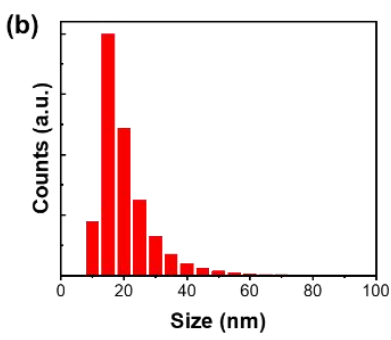

DSPE-PEG capped SMILES NP

(c)

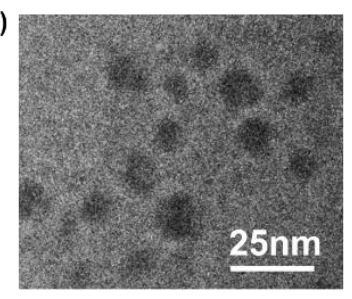

Figure 4. (a) Schematic illustration of the preparation of DSPE-PEG capped SMILES nanoparticles through nanoprecipitation. (b) Hydrodynamic size distribution histogram of SMILES nanoparticles (with 2.5 eq CS) capped with 11 $w \%$ DSPE-PEG acquired from DLS measurements. (c) Cryo-TEM image of SMILES nanoparticles (with 2.5 eq CS) capped with 11 wt\% DSPE-PEG.

\section{Stabilizing SMILES Nanoparticles by Capping with Polyethylene glycol Amphiphiles.}

The high fluorescence quantum yield and brightness of SMILES nanoparticles make bioimaging an obvious potential application. However, for bioimaging and many other applications, it is essential to cap the particles with amphiphilic materials to improve their colloidal stability and biocompatibility in aqueous environments.

To cap the SMILES nanoparticles we use the polyethylene glycol based detergent 1,2-distearoyl-sn-glycero-3phosphatidylethanolamine- $N$-[methoxy(polyethylene glycol) (DSPE-PEG). ${ }^{[22]}$ Derivatives of DSPE-PEG are ideal capping agents for the surface of hydrophobic nanoparticles and produce excellent colloidal stability. ${ }^{[23]}$ It has also been reported that surface capping agents play an important role in the photophysical properties of fluorescent nanoparticles, ${ }^{[20 \mathrm{a}]}$ especially as they can reduce surface trap states and thus enhance fluorescence. ${ }^{[24]}$ In the case of SMILES nanoparticles of size $\sim 20 \mathrm{~nm}$ composed of dyes separated by stacks of 2:1 cyanostar-anion complexes (Fig. 1), there are about 10 of these repeat units across the center of the particle. Of the $\sim 800$ dyes within such a nanoparticle, approximately half of them would be located in the outermost 2-nm shell and would be more susceptible to surface quenching. Thus, DSPE-PEG may suppress some of these surface states. Furthermore, DSPE-PEG is reported to increase systemic circulation time of nanoparticles in animals, prohibit nanoparticle agglomeration, and reduce the toxicity of nanoparticles for in vivo applications. ${ }^{[25]}$

The surface-capped SMILES nanoparticles were prepared simply by adding the DSPE-PEG to the THF stock solutions used in the aqueous nanoprecipitation (Fig. 4a). The obtained SMILES nanoparticles that are capped with DSPE-PEG are smaller and show a narrower size distribution (16 $\pm 5 \mathrm{~nm}$, Fig. 4b,c and S8) relative to the neat particles made without the capping agent $(25$ $\pm 8 \mathrm{~nm}$, Fig $2 \mathrm{~b}$ and S2). This finding agrees with previous reports in which small nanoparticles were prepared using DSPE-PEG derivatives as the surface capping agent. ${ }^{[5,23,26]}$ The colloidal stability of SMILES nanoparticles is also improved by capping them with DSPE-PEG. The nanoparticles made without surface capping agent show agglomeration over time with precipitation occurring over the course of a few days. The DSPE-PEG capped SMILES nanoparticles however show colloidal stability and a stable size distribution for more than four weeks.

The amount of DSPE-PEG used in the precursor solutions composed of R12 and 2.5 molar equivalents of cyanostar was systematically varied from 6 to $66 w \%$. Across this range, we saw an initial increase in the fluorescent quantum yield of the resulting SMILES nanoparticles from $\phi=0.25$ without DSPE-PEG to $\phi=$ 0.30 with 11 wt\% of DSPE-PEG, indicating passivation of the particle surface. Beyond $11 w t \%$ no further improvements were observed (Table S5). Analysis of the nanoparticles made using 11 and $66 w t \%$ DSPE-PEG showed very similar sizes and size distributions seen by DLS and cryo-TEM (Fig. S8). Therefore, excess DSPE-PEG has little impact on the structure of the nanoparticles. The observation that excess DSPE-PEG does not alter the fluorescence quantum yield or particle size reveals that the amphiphiles are only covering the surface of the particles and are not diluting the core of the SMILES nanoparticles (Fig. 4a). This encouraging result suggests that the structures and photophysics of the SMILES core can be predictably designed and tuned according to the supramolecular principles behind SMILES (anion binding and charge-by-charge assembly), independently from the surface coating.

The optimized rhodamine R12 SMILES nanoparticles coated with DSPE-PEG (11 wt\%) and made with 2.5 molar equivalents of cyanostar reach a fluorescence quantum yield of $30 \%$ and a volume-normalized brightness, $B / V$, of over $5000 \mathrm{M}^{-1} \mathrm{~cm}^{-1} / \mathrm{nm}^{3}$ by direct excitation $\left(\lambda_{\mathrm{ex}}=560 \mathrm{~nm}\right)$ of the R12 dye and $6600 \mathrm{M}^{-1}$ $\mathrm{cm}^{-1} / \mathrm{nm}^{3}$ for UV excitation $\left(\lambda_{\mathrm{ex}}=320 \mathrm{~nm}\right.$ ) of the cyanostar macrocycles. These resulting brightnesses are the highest reported among organic fluorescent nanoparticles derived from molecular dyes. ${ }^{[5]}$ 
(a)

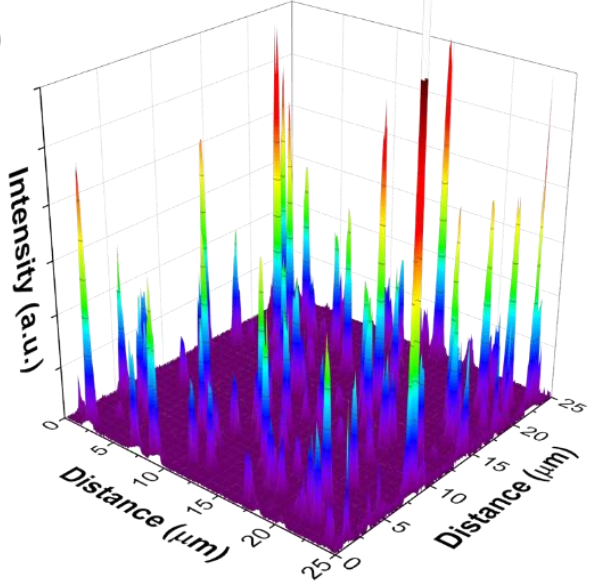

(b)

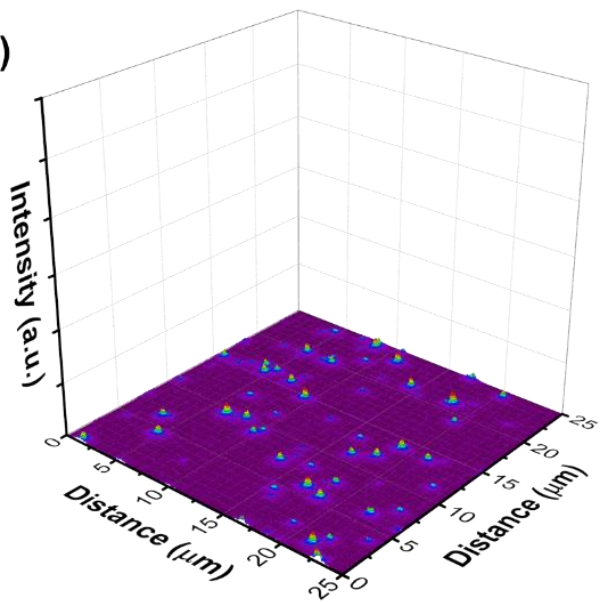

Figure 5. Single particle fluorescence microscopy images of SMILES R12 nanoparticles with 2.5 eq CS (a) and FluoSphere ${ }^{\circledR}$ NPs (b) measured under identical condition, the fluorescence intensities are relative intensity corrected for size of the particles.

\section{Comparative Evaluation of the Brightness of SMILES Nanoparticles.}

The brightness of single SMILES nanoparticles was compared to the commercially available $40-\mathrm{nm}$ FluoSpheres ${ }^{\circledR}$ particles with nearly identical spectral properties (Fig. S9). The fluorescence intensity from single particles prepared at high dilution by deposition onto a glass surface was measured using confocal fluorescence microscopy (Fig. 5). SMILES nanoparticles are clearly much brighter than the FluoSpheres ${ }^{\circledR}$ measured under identical conditions. This difference is associated with the almost 20 times greater dye density in SMILES nanoparticles compared with the FluoSpheres ${ }^{\circledR}$ (Table S6). The fluorescence spectra of 10 randomly selected single SMILES nanoparticles overlap with each other (Fig. S10a), which indicates their spectral homogeneity and ensures a narrow emission spectrum (Fig. 2d). Each of the single-particle fluorescence decays display similar lifetimes (see Table S7 and Fig. S10b). However, and unlike the FluoSpheres ${ }^{\circledR}$ with their single-exponential fluorescence decays, the SMILES nanoparticles require a double-exponential function to fit the fluorescence decay, indicating internal heterogeneity in the non-radiative rates within SMILES nanoparticles. We assign this outcome to variable rates of energy migration to different quenching sites depending on their location within the nanoparticles, which is a common feature of organic nanoparticles with high dye loadings. ${ }^{[16 a, 20 b]}$

Reisch, Klymchenko and co-workers optimized organic fluorescent nanoparticles by loading a lipophilic rhodamine dye that is paired with bulky fluorinated counterions into polymer nanoparticles. ${ }^{[5,16 a, 18,20 \mathrm{~b}]}$ In the very best case, their particles reached a volume-normalized brightness of $B / V \sim 4700 \mathrm{M}^{-1} \mathrm{~cm}^{-}$ $1 / \mathrm{nm}^{3}\left(\phi=34 \%, B=8.9 \times 10^{7} \mathrm{M}^{-1} \mathrm{~cm}^{-1}\right.$, size $\left.=33 \mathrm{~nm}\right) .{ }^{[16 \mathrm{a}]}$ Nanoparticles based on aggregation induced emission, can reach a high dye loading, but the absorption coefficients of the dyes $(\varepsilon$ in the range of $10000-30000 \mathrm{M}^{-1} \mathrm{~cm}^{-1}$ ) limit their $B / V$ values to approximately $1000 \mathrm{M}^{-1} \mathrm{~cm}^{-1} \mathrm{~nm}^{-3}$. ${ }^{[5]}$ For comparison, the normalized brightness of the first samples of SMILES nanoparticles (2.5 eq. CS, 11 wt\% DSPE-PEG) exceeded 5000 $\mathrm{M}^{-1} \mathrm{~cm}^{-1} / \mathrm{nm}^{3}(\phi=30 \%)$ and are all expressed in a very attractive small size $(16 \mathrm{~nm})$.
The optical properties of the SMILES nanoparticles are remarkably similar to those obtained from the SMILES crystals ${ }^{[17]}$ based on the rhodamine $3 \mathrm{~B}$ dye despite their non-crystalline structure, the presence of the capping agent, and the nanometric size of the nanoparticles. This similarity confirms the robustness of the hierarchical SMILES self-assembly process that can reliably deliver charge-by-charge packing of dyes without the introduction of additional defects to deteriorate the performance. Therefore, SMILES have the ability to deliver predictable optical properties and high fluorescence brightness in both macroscale and nanoscale materials.

\section{Live Cell Imaging with SMILES Nanoparticles.}

The very high brightness and full organic nature of SMILES nanoparticles make them promising fluorescent markers for bioimaging. To perform an initial investigation into the applicability of SMILES nanoparticles for imaging in biological media, live HEK 293 cells were incubated for 4 hours at $37^{\circ} \mathrm{C}$ with SMILES nanoparticles (2.5 eq CS, 66 wt\% DSPE-PEG). As a control experiment, HEK 293 cells were incubated with $\mathbf{R} 12$ aggregates (capped with 66 wt\% DSPE-PEG but formed without any cyanostar). In both cases, the cells were extensively washed before imaging by confocal fluorescence microscopy. When using SMILES nanoparticles, clusters of bright intensities were observed inside the cells while none were observed along the cell membranes, which indicates that the DSPE-PEG capped SMILES nanoparticles enter inside the live cells (Fig 6). In the case of the R12 aggregates, no fluorescence emission signal was detected under the same experimental conditions (Fig. 6) on account of their much lower brightness $\left(B / V \sim 800 \mathrm{M}^{-1} \mathrm{~cm}^{-1} /\right.$ $\left.\mathrm{nm}^{3}\right)$. 
The location of DSPE-PEG capped SMILES nanoparticles inside the cells was investigated using three commercially available cell stains (see Section S8): cytosolic dye Calcein AM, nuclear dye NucBlue, and Mitotracker-Green. Calcein is a membrane permeable dye that only displays fluorescence after being cleaved by an intracellular enzyme. Thus, the labeling of all the cells containing SMILES nanoparticles with calcein indicates good viability of the cells and the absence of toxicity of the nanoparticles after several hours of incubation. Mitotracker is a cell reagent that specifically labels mitochondria. Co-localization of the fluorescent signals from SMILES nanoparticles and Mitotracker indicates that the particles are primarily localized in or at the surface of mitochondria.
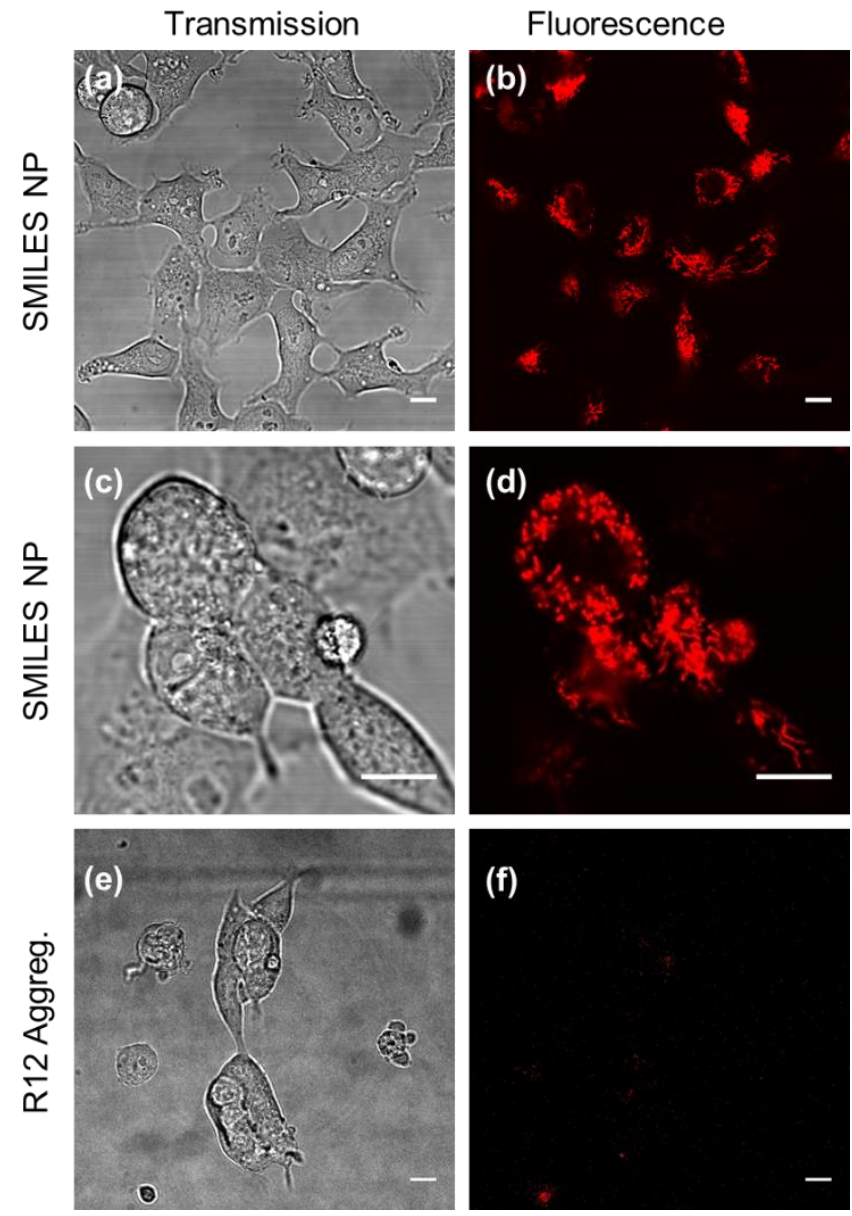

Figure 6. Imaging of HEK 293 cells incubated for 4 hours with either surfacecapped (66 wt\% DSPE-PEG) SMILES nanoparticles (2.5 eq. CS) or surfacecapped (66 wt\% DSPE-PEG) R12 aggregates as a control. (a, c, and e) Transmission channels from micrograph in the presence SMILES nanoparticles or R12 aggregates. (b, d, and f) Fluorescence channels of SMILES nanoparticles (excitation at $543 \mathrm{~nm}$ ) at low (b) and high magnification (d). (f) Fluorescence channel of $\mathbf{R} 12$ aggregates. Scale bars, $10 \mu \mathrm{m}$.

\section{Conclusion}

We report the first successful translation of the high brightness and predictable optical properties of SMILES materials from macroscopic crystals to nanoparticles with preservation of the supramolecular structure and the attractive optical properties. A simple one-step nano-precipitation method was developed yielding either neat SMILES nanoparticles or SMILES nanoparticles bearing a stabilizing PEG-based coating. The
SMILES concept allows small nanoparticles to be formulated in which high dye densities are produced without suffering from aggregation caused quenching. In this way, the spectral properties of the dye in dilute solution are reproduced and their very high intrinsic brightness can be used to produce ultra-bright nanoparticles. The volume-normalized brightness of SMILES nanoparticles is only slightly less than that the record-breaking brightness discovered in macroscopic single crystals of SMILES materials and found to be on par with the highest values reported for dye-based nanoparticles. This outstanding optical behavior can be achieved as a result of the robustness of the self-assembly process that is driven by the structure-directing properties of the anion-binding cyanostar receptors. Furthermore, we find that the cyanostar serves as a UV antenna system endowing SMILES nanoparticles with the additional feature of very high brightness upon UV excitation. Bioimaging applications were confirmed by imaging living mammalian cells labeled with surfactant-capped SMILES nanoparticles. The simple approach opens up a new way to develop bright and full-organic fluorescent nanoparticles with both tunable and predictable properties for optical applications by using the general concept of SMILES materials.

\section{Acknowledgements}

This work was supported by the Danish Council of Independent Research (DFF - 0136-00122B), the Lundbeck Foundation (grant R303-2018-3237), the National Science Foundation (DMR1533988), and Bridge Funding support from the Office of the Vice Provost for Research, the College of Arts and Sciences, and the Department of Chemistry at Indiana University.

Keywords: SMILES • fluorescent nanoparticles • fluorescent dyes $\cdot$ cell imaging $\bullet$ cyanostar macrocycles

[1] a) Z. Chen, C. L. Ho, L. Wang, W. Y. Wong, Adv. Mater. 2020, 32, 1903269 ; b) S. D. Xu, Y. K. Duan, B. Liu, Adv. Mater. 2020, 32, 1903530; c) O. S. Wolfbeis, Chem. Soc. Rev. 2015, 44, 4743-4768; d) Y. Shu, X. Lin, H. Qin, Z. Hu, Y. Jin, X. Peng, Angew. Chem., Int. Ed. Engl. 2020, 59, 2231222323; e) H. Zhu, J. L. Fan, J. J. Du, X. J. Peng, Acc. Chem. Res. 2016, 49, 2115-2126; f) E. Middha, B. Liu, ACS Nano 2020, 14, 9228-9242.

[2] P. Hajeb, J. J. Sloth, S. Shakibazadeh, N. A. Mahyudin, L. Afsah-Hejri, Compr. Rev. Food Sci. Food Saf. 2014, 13, 457-472.

[3] a) Y. F. Huang, Y. B. Zhang, F. J. Huo, Y. Wen, C. X. Yin, Sci. China Chem. 2020, 63, 1742-1755; b) J. V. Jun, D. M. Chenoweth, E. J. Petersson, Org. Biomol. Chem. 2020, 18, 5747-5763.

[4] a) S. Sindhwani, A. M. Syed, J. Ngai, B. R. Kingston, L. Maiorino, J. Rothschild, P. MacMillan, Y. W. Zhang, N. U. Rajesh, T. Hoang, J. L. Y. Wu, S. Wilhelm, A. Zilman, S. Gadde, A. Sulaiman, B. Ouyang, Z. Lin, L. S. Wang, M. Egeblad, W. C. W. Chan, Nat. Mater. 2020, 19, 566-575; b) J. L. Perry, K. G. Reuter, J. C. Luft, C. V. Pecot, W. Zamboni, J. M. DeSimone, Nano Lett. 2017, 17, 2879-2886; c) X. K. Sun, R. Rossin, J. L. Turner, M. L. Becker, M. J. Joralemon, M. J. Welch, K. L. Wooley, Biomacromolecules 2005, 6, 2541 2554.

[5] A. Reisch, A. S. Klymchenko, Small 2016, 12, 1968-1992. 
[6] a) J. Cornil, D. Beljonne, J. P. Calbert, J. L. Bredas, Adv. Mater. 2001, 13, 1053-1067; b) M. Kasha, H. R. Rawls, M. A. El-Bayoumi, Pure Appl. Chem. 1965, 11, 371-392; c) J. B. Birks, Photophysics of Aromatic Molecules, Wiley: London, 1970, pp 15- 20.; d) Y. J. Huang, J. Xing, Q. Y. Gong, L. C. Chen, G. F. Liu, C. J. Yao, Z. R. Wang, H. L. Zhang, Z. Chen, Q. C. Zhang, Nat. Commun. 2019, 10, 169.

[7] M. J. Chen, M. Z. Yin, Prog. Polym. Sci. 2014, 39, 365-395.

[8] a) R. Meallet-Renault, A. Herault, J. J. Vachon, R. B. Pansu, S. Amigoni-Gerbier, C. Larpent, Photoch. Photobio. Sci. 2006, 5, 300-310; b) K. Trofymchuk, A. Reisch, I. Shulov, Y. Mely, A. S. Klymchenko, Nanoscale 2014, 6, 12934-12942; c) X. Zhang, Z. J. Chen, F. Wurthner, J. Am. Chem. Soc. 2007, 129, 4886-4887; d) V. Caponetti, J. W. Trzcinski, A. Cantelli, R. Tavano, E. Papini, F. Mancin, M. Montalti, Front. Chem. 2019, 7, 168.

[9] a) C. F. Wu, T. Schneider, M. Zeigler, J. B. Yu, P. G. Schiro, D. R. Burnham, J. D. McNeill, D. T. Chiu, J. Am. Chem. Soc. 2010, 132, 1541015417; b) C. Grazon, J. Rieger, R. Meallet-Renault, B. Charleux, G. Clavier, Macromolecules 2013, 46, 5167-5176; c) Z. Tian, A. D. Shaller, A. D. Li, Chem. Commun. 2009, 2, 180-182.

[10] a) K. M. L. Taylor-Pashow, J. Della Rocca, Z. G. Xie, S. Tran, W. B. Lin, J. Am. Chem. Soc. 2009, 131, 14261-14263; b) D. M. Liu, R. C. Huxford, W. B. Lin, Angew. Chem., Int. Ed. Engl. 2011, 50, 3696-3700; c) W. Cai, H. Y. Gao, C. C. Chu, X. Y. Wang, J. Q. Wang, P. F. Zhang, G. Lin, W. G. Li, G. Liu, X. Y. Chen, ACS Appl. Mater. Interfaces 2017, 9, 2040-2051.

[11] K. Cui, X. M. Lu, W. Cui, J. Wu, X. M. Chen, Q. H. Lu, Chem. Commun. 2011, 47, 920-922.

[12] J. G. Woller, J. K. Hannestad, B. Albinsson, J. Am. Chem. Soc. 2013, 135, 2759-2768.

[13] W. Chen, C. A. Cheng, E. D. Cosco, S. Ramakrishnan, J. G. P. Lingg, O. T. Bruns, J. I. Zink, E. M. Sletten, J. Am. Chem. Soc. 2019, 141, 1247512480 .

[14] a) J. D. Luo, Z. L. Xie, J. W. Y. Lam, L. Cheng, H. Y. Chen, C. F. Qiu, H. S. Kwok, X. W. Zhan, Y. Q. Liu, D. B. Zhu, B. Z. Tang, Chem. Commun. 2001, 18, 1740-1741; b) X. G. Gu, X. Y. Zhang, H. L. Ma, S. R. Jia, P. F. Zhang, Y. J. Zhao, Q. Liu, J. G. Wang, X. Y. Zheng, J. W. Y. Lam, D. Ding, B. Z. Tang, Adv. Mater. 2018, 30, 1801065; c) I. O. Aparin, N. Melnychuk, A. S. Klymchenko, Adv. Opt. Mater. 2020, 8, 2000027; d) X. L. Cai, B. Liu, Angew. Chem., Int. Ed. Engl. 2020, 59, 9868-9886.

[15] F. Würthner, Angew. Chem., Int. Ed. Engl. 2020, 59, 14192-14196.
[16] a) A. Reisch, K. Trofymchuk, A. Runser, G. Fleith, M. Rawiso, A. S. Klymchenko, ACS Appl. Mater. Interfaces 2017, 9, 43030-43042; b) A. Reisch D. Heimburger, P. Ernst, A. Runser, P. Didier, D. Dujardin, A. S. Klymchenko, Adv. Funct. Mater. 2018, 28, 1805157; c) N. Melnychuk, A. S. Klymchenko, J. Am. Chem. Soc. 2018, 140, 10856-10865; d) K. Trofymchuk, A. Reisch, P. Didier, F. Fras, P. Gilliot, Y. Mely, A. S. Klymchenko, Nat Photonics 2017, 11, 657-663; e) B. Andreiuk, A. Reisch, M. Lindecker, G. Follain, N. Peyrieras, J. G. Goetz, A. S. Klymchenko, Small 2017, 13, 1701582; f) I. Khalin, D. Heimburger, N. Melnychuk, M. Collot, B. Groschup, F. Hellal, A. Reisch, N. Plesnila, A. S. Klymchenko, ACS Nano 2020, 14, 9755-9770; g) M. Collot, J. Schild, K. T. Fam, R. Bouchaala, A. S. Klymchenko, ACS Nano 2020, 14, 13924-13937.

[17] C. R. Benson, L. Kacenauskaite, K. L. VanDenburgh, W. Zhao, B. Qiao, T. Sadhukhan, M. Pink, J. S. Chen, S. Borgi, C. H. Chen, B. J. Davis, Y. C. Simon, K. Raghavachari, B. W. Laursen, A. H. Flood, Chem 2020, 6, 19781997.

[18] I. Shulov, S. Oncul, A. Reisch, Y. Arntz, M. Collot, Y. Mely, A. S. Klymchenko, Nanoscale 2015, 7, 18198-18210.

[19] B. W. Laursen, J. Reynisson, K. V. Mikkelsen, K. Bechgaard, N. Harrit, Photoch. Photobio. Sci. 2005, 4, 568-576.

[20] a) B. Yang, J. Chen, F. Hong, X. Mao, K. Zheng, S. Yang, Y. Li, T. Pullerits, W. Deng, K. Han, Angew. Chem., Int. Ed. Engl. 2017, 56, 12471 12475; b) A. Reisch, P. Didier, L. Richert, S. Oncul, Y. Arntz, Y. Mely, A. S. Klymchenko, Nat. Commun. 2014, 5, 4089.

[21] a) E. M. Fatila, E. B. Twum, J. A. Karty, A. H. Flood, Chem.-Eur. J. 2017, 23, 10652-10662; b) E. M. Fatila, M. Pink, E. B. Twum, J. A. Karty, A. H. Flood, Chem. Sci. 2018, 9, 2863-2872.

[22] L. Vukovic, F. A. Khatib, S. P. Drake, A. Madriaga, K. S. Brandenburg, P. Kral, H. Onyuksel, J. Am. Chem. Soc. 2011, 133, 13481-13488.

[23] K. Li, B. Liu, Chem. Soc. Rev. 2014, 43, 6570-6597.

[24] a) C. Carrillo-Carrion, S. Cardenas, B. M. Simonet, M. Valcarcel, Chem Commun. 2009, 35, 5214-5226; b) L. Xu, K. J. Chen, H. M. El-Khair, M. H. Li, X. F. Huang, Appl. Surf. Sci. 2001, 172, 84-88.

[25] J. S. Suk, Q. G. Xu, N. Kim, J. Hanes, L. M. Ensign, Adv. Drug Delivery Rev. 2016, 99, 28-51.

[26] G. X. Feng, K. Li, J. Liu, D. Ding, B. Liu, Small 2014, 10, 1212-1219. 


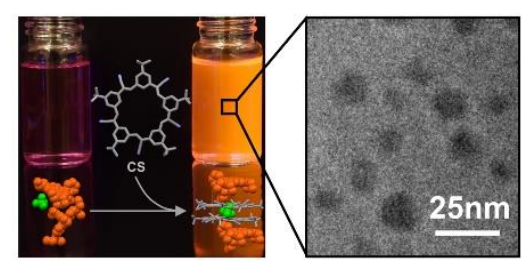

Ultra-bright nanoparticles are prepared in a simple one-step supramolecular self-assembly approach by nanoprecipitation of fluorescent rhodamine dye with macrocyclic anion receptor. Binding of the dye's counter ion by the cyanostar macrocycle ensures separation of dyes to prohibit self-quenching while also acting as UV antenna system to boost particle fluorescence. 16-nm glycolstabilized nanoparticles are used for live cell imaging. 